\title{
A New Synergistic Forecasting Method for Short-Term Traffic Flow with Event-Triggered Strong Fluctuation
}

\author{
Darong Huang $(\mathbb{D}$, Zhenping Deng $(\mathbb{D}$, and Bo Mi \\ Institute of Information Science and Engineering, Chongqing Jiaotong University, Chongqing 400074, China \\ Correspondence should be addressed to Darong Huang; drhuang@cqjtu.edu.cn
}

Received 22 December 2017; Accepted 12 February 2018; Published 19 March 2018

Academic Editor: Zhijie Zhou

Copyright (C) 2018 Darong Huang et al. This is an open access article distributed under the Creative Commons Attribution License, which permits unrestricted use, distribution, and reproduction in any medium, provided the original work is properly cited.

\begin{abstract}
Directing against the shortcoming of low accuracy in short-term traffic flow prediction caused by strong traffic flow fluctuation, a novel method for short-term traffic forecasting based on the combination of improved grey Verhulst prediction algorithm and first-order difference exponential smoothing is proposed. Firstly, we constructed an improved grey Verhulst prediction model by introducing the Markov chain to its traditional version. Then, based on an introduced dynamic weighting factor, the improved grey Verhulst prediction method, and the first-order difference exponential smoothing technique, the new method for short-term traffic forecasting is completed in an efficient way. Finally, experiment and analysis are carried out in the light of actual data gathered from strong fluctuation environment to verify the effectiveness and rationality of our proposed scheme.
\end{abstract}

\section{Introduction}

In recent years, the popularization of seamless links among heterogeneous traffic equipment brought about higher requirements on the real-time and reliability of short-term traffic flow prediction. With continuous improvement of traffic information processing, how to predict the short-term traffic flow accurately and effectively has aroused wide attention of scholars domestically and abroad [1-3], whereupon numerous of prominent research results have emerged. So far, relevant academic circles mainly focus on the construction and optimization of prediction models in terms of time series, linear regression, historical average model, Kalman filtering, grey theory, chaos theory, nonparametric regression, neural network, support vector machine, dynamic traffic assignment model, and so forth [4-9]. These algorithms and models mentioned above are relatively mature, and their prediction effects are acceptable under the environment of favorable traffic flow stability. However, once the traffic data are seriously fluctuated, one single model can neither guarantee the prediction accuracy nor break through certain limitations of operations to predict short-term traffic flow under the environments of heterogeneous traffic equipment transmission.

In order to solve the aforementioned problems, domestic and foreign scholars have contrived an improved model to realize fusion prediction with the advantages of different models integrated of short-term traffic flow. For example, Xie et al. [10] improved the search efficiency of $K$-nearest neighbor algorithm by cooperating the multivariate statistical regression model and the pattern distance search method based on the analysis of original $K$-nearest neighbor algorithm for short-term traffic flow prediction. Their experimental results indicated that the prediction effect is better when the $K$ values are reasonable. Fan et al. [4] exploited the characteristics of time-varying and nonlinearity of traffic flow and proposed a new hybrid forecasting model based on the nonparametric regression model and the BP neural network model; they also employed fuzzy control to determine the weight of each single model. However, the basic support platform of the hybrid model is the traffic flow database, so the demand of data volume is large. Once the data volume is insufficient, the prediction accuracy will be depressed. Xiao et al. [11] proposed an improved binding cycle truncation accumulated generating operation seasonal grey rolling forecasting model based on the properties of similar seasonality within intraday and weekly trends. The model weakens the random disturbance and highlights the intrinsic grey exponent rule after accumulating the sequence, so that the model has better performance under different traffic flow conditions. Lin-chao et al. [12] proposed a short-term traffic flow prediction model 
based on support vector regression which is suitable for realtime monitoring and then analyzed the model parameters by grid search under the premise of considering the influence of space-time factors. The model can achieve ideal prediction effect even if the real-time traffic data are insufficient. Nevertheless, the accuracy of the model will decrease when the traffic status changes. In order to solve such problem, Ma et al. [13] proposed a two-dimensional prediction method by using the Kalman filtering theory based on historical data. The advantage of this method is that the two predicted values are fused by using an equation with weight coefficients where the weight coefficients can be generated in real time in the process of prediction. Experimental results show that the model has an admirable predictive effect. Chan et al. [14] used exponential smoothing to preprocess traffic data that is taken as the input of network and then used Levenberg Marquardt (LM) variant algorithm to train the network weights, making the generalization ability of network enhanced.

However, thanks to the diversified developments of traffic information processing and data transmission techniques within heterogeneous traffic network, as well as the impact caused by dynamic changes of road topology, traffic accidents, severe weather, driving styles, and so forth, short-term traffic data are instantaneous and the irregular volatility is always changing $[15,16]$. In this case, the above combinational algorithm can just solve the short-term traffic prediction problem in certain circumstance, and the prediction effect can hardly be achieved in the light of the real-time requirement. Hence, it is urgent to construct new models and algorithms to deal with the short-term traffic forecasting problem aiming at the strong fluctuation triggered by events. Thus, in this paper, we intend to propose a novel method for short-term traffic prediction based on the energetically grey Verhulst prediction algorithm and the first-order difference exponential smoothing technique to solve the problem of low prediction accuracy caused by traffic event-triggering strong fluctuation.

The layout of this paper is arranged as follows. Firstly, based on the introduction of the traditional grey Verhulst model, an improved grey Markov forecasting model is devised by introducing Markov chain. Secondly, combining the advantages of forecasting by utilizing first-order difference exponential smoothing algorithm and introducing a dynamic weighting factor, a new method for short-term traffic forecasting is concretely constructed according to the afore contrived models. Finally, comparative analysis of the examples illustrated that our proposed model and algorithm are more effective.

\section{Short-Term Traffic Flow Prediction Model Based on Grey Difference Exponential Smoothing}

\subsection{Short-Term Traffic Flow Prediction Model Based on Grey Markov Theory}

2.1.1. Introduction of Grey Verhulst Model. The grey system theory was first put forward by Professor Deng Julong, a Chinese scholar, in 1980s [17]. Its quantitative model is mainly based on the structure of number generating, which makes the prediction effect no longer affected by the empirical statistical law gained from the analysis of mass data. At the same time, it surmounted the limitation of white system and the black system relying solely on the probability and statistics method. Therefore, the grey system theory has been widely used in the fields of agricultural production, industrial control, traffic management, and so forth [18].

Classical grey system theory mainly includes GM $(1,1)$ model, GM $(2,1)$ model, grey Verhulst model, and so forth [19]. Considering the strong randomness and nonlinearity of traffic flow with strong fluctuation in heterogeneous information model, the data sequence significantly gives expression to the characteristics of nonmonotonic oscillatory development or the S-shaped property of saturation trend, which makes the grey GM $(1,1)$ prediction model unsuitable. Therefore, we choose the grey Verhulst model in this paper to forecast short-term traffic. The basic modeling process is described as follows.

The nonnegative data sequence is defined as

$$
x^{(0)}=\left(x_{1}{ }^{(0)}, x_{2}{ }^{(0)}, \ldots, x_{n}{ }^{(0)}\right) .
$$

while $x^{(1)}$ stands for the one-time accumulation sequence (1Ago):

$$
\begin{aligned}
x^{(1)} & =\left(x_{1}{ }^{(1)}, x_{2}{ }^{(1)}, \ldots, x_{n}{ }^{(1)}\right), \\
x_{k}{ }^{(1)} & =\sum_{i=1}^{k} x_{i}{ }^{(0)} \quad(k=1,2, \ldots, n) .
\end{aligned}
$$

Then, assuming that $z^{(1)}$ represents the consecutive neighbor sequence of $x^{(1)}$,

$$
\begin{aligned}
z^{(1)} & =\left(z_{1}^{(1)}, z_{2}^{(1)}, \ldots, z_{n}^{(1)}\right), \\
z_{k}^{(1)} & =0.5\left(x_{k}^{(1)}+x_{k-1}{ }^{(1)}\right) \quad(k=2,3, \ldots, n) .
\end{aligned}
$$

The grey Verhulst model and its whitening equation can be defined as follows.

Definition 1 (see $[20,21])$. The grey Verhulst model is

$$
x^{(0)}+a z^{(1)}=b\left(z^{(1)}\right)^{2},
$$

where $a$ and $b$ are the parameters of the equation.

Definition 2 (see $[20,21])$. The whitening equation of the grey Verhulst model is

$$
\frac{d x^{(1)}}{d t}+a x^{(1)}=b\left(x^{(1)}\right)^{2},
$$

where $a, b$, and $t$ are the parameters of the whitening equation.

As deduced in $[20,21]$, the following conclusions are drawn. 
Theorem 3. If the grey Verhulst model is defined as formula (4) and $\hat{a}=(a, b)^{T}$ represents the parameter column, let

$$
\begin{aligned}
& B=\left[\begin{array}{cc}
-z_{2}{ }^{(1)} & \left(z_{2}{ }^{(1)}\right)^{2} \\
-z_{3}{ }^{(1)} & \left(z_{3}^{(1)}\right)^{2} \\
\vdots & \vdots \\
-z_{n}{ }^{(1)} & \left(z_{n}^{(1)}\right)^{2}
\end{array}\right], \\
& Y=\left[\begin{array}{c}
x_{2}^{(0)} \\
x_{3}^{(0)} \\
\vdots \\
x_{n}{ }^{(0)}
\end{array}\right]
\end{aligned}
$$
fies

The least squares estimator of the parameter column satis-

$$
\widehat{\alpha}=\left(B^{T} B\right)^{-1} B^{T} Y
$$

Theorem 4. If the grey Verhulst model is defined as definition (2), the solution of the whitening equation can be deduced as

$$
x^{(1)}(t)=\frac{a x_{0}{ }^{(1)}}{b x_{0}{ }^{(1)}+\left(a-b x_{0}{ }^{(1)}\right) e^{a t}} .
$$

According to Theorems 3 and 4, the following conclusions are drawn.

Inference 5. The time response sequence of grey Verhulst model can be defined as

$$
\widehat{x}_{k+1}^{(1)}=\frac{a x_{0}^{(1)}}{b x_{0}^{(1)}+\left(a-b_{0}^{(1)}\right) e^{a t}}, \quad(k=1,2, \ldots, n) .
$$

Let $x_{0}{ }^{(1)}=x_{1}{ }^{(0)}$; we iterate formula (9) to be

$$
\widehat{x}_{k+1}{ }^{(1)}=\frac{a x_{1}{ }^{(0)}}{b x_{1}{ }^{(0)}+\left(a-b x_{1}{ }^{(0)}\right) e^{a t}}, \quad(k=1,2, \ldots, n) .
$$

The reduction formula can then be defined as

$$
\widehat{x}_{k+1}{ }^{(0)}=\widehat{x}_{k+1}{ }^{(1)}-\widehat{x}_{k}{ }^{(1)}, \quad(k=1,2, \ldots, n) .
$$

Pointing at the traffic flow with the trend of increasing saturation, numerous traffic prediction algorithms have been proposed taking advantage of the grey Verhulst model to achieve preferable prediction effects. However, as a complicated nonlinear system involving multitudinous uncertainties, the probability of fortuitous events on urban roads is highly fluctuant, which leads to the deviation of forecasting results. Therefore, it is necessary to take the nonlinearity and time-varying characteristics of overall interactions into account in line with various influential factors. So, we improve the algorithm aiming at the accuracy of prediction model next.
2.1.2. Short-Term Traffic Flow Prediction Model Based on Grey Markov Theory. During the process of traffic data aggregation, which is coordinated by complex human-vehicleenvironment interaction, the current traffic flow is often affected by previous moments. Therefore, in order to improve the prediction accuracy of grey Verhulst model, mathematical description of traffic flow aggregation is given in advance by constructing the Markov state transition probability matrix [22].

It is obvious that the state transition probability matrix should be updated over time when Markov is used to optimize the grey model. That is to say, at time point $t+1$, the state transition probability of Markov chain should be recalculated according to real-time traffic data along with the state transition probability matrix updated synchronously. Therefore, at time point $t+1$, the probability of traffic flow aggregation from state $i$ to $j$ can be described as

$$
P_{i j}=p\left(X_{t+1}=j \mid X_{t}=i\right) .
$$

And the updated Markov transition probability matrix is formulated to be

$$
P=\left[\begin{array}{cccc}
p_{11} & p_{12} & \cdots & p_{1 n} \\
p_{21} & p_{22} & \cdots & p_{2 n} \\
\vdots & \vdots & & \vdots \\
p_{n 1} & p_{n 2} & \cdots & p_{n n}
\end{array}\right],
$$

where each element $p_{i j}$ is constrained as $p_{i j} \geq 0, i, j \in I$, and $\sum_{j \in I} p_{i j}=1, i \in I$.

The grey Markov forecasting model is shown in Figure 1. According to the flow chart of this model, the value of grey Verhulst model to be predicted is $\widehat{x}$ at time point $t+1$. In order to make the predicted value as close to the true value as possible, the true value at the time point $t$ is utilized and represented as $x_{i j}$, and the transition probability from the true value of time point $t$ to the predictive value is denoted by $p_{i j}$. Thus, the cumulative sum of the product of the traffic flow average value and its corresponding probability from the current state to other states can be taken as the predicted value of the grey Markov model:

$$
G_{t+1}= \begin{cases}\hat{x}+\phi & \left(p_{i j}=0\right) \\ \hat{x} \cdot p_{i j}+\phi+\sum_{k=1, k \neq j}^{n} p_{i k} \cdot \bar{x}_{i k} & \left(p_{i j} \neq 0\right),\end{cases}
$$

where $\phi$ is the adjustment factor and $\phi=\widehat{x}_{t}-\widehat{x}_{t-1}$.

The adjustment factor $\phi$ is used to solve the data fluctuation problem of collaborative traffic flow prediction in terms of human, vehicle, and environment interaction. However, the smoothness of short-term traffic flow forecasting remains to be improved once events trigger strong fluctuations caused by traffic flow aggregation. Therefore, in order to continuously improve the prediction smoothness together with accuracy, a grey difference exponential smoothing short-term traffic prediction method is further constructed by introducing the first-order difference exponential smoothing model in the next subsection. 


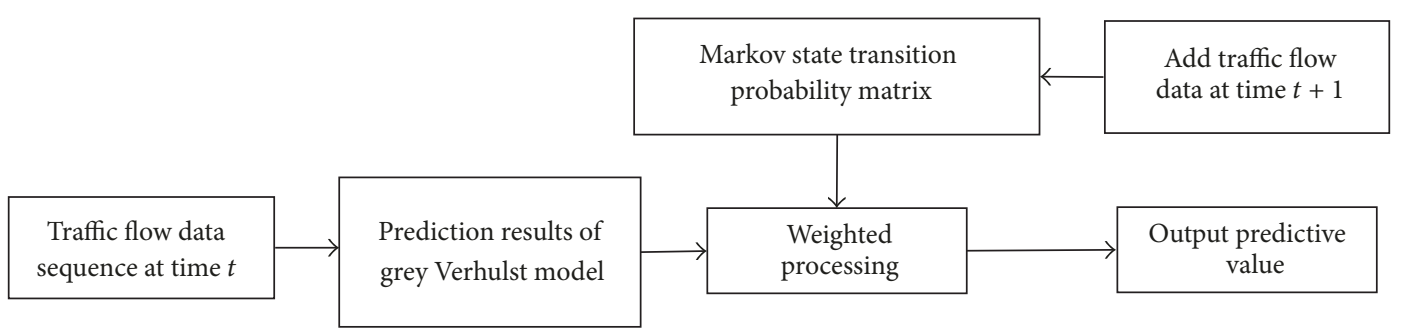

FIGURE 1: Grey forecasting model based on Markov chain.

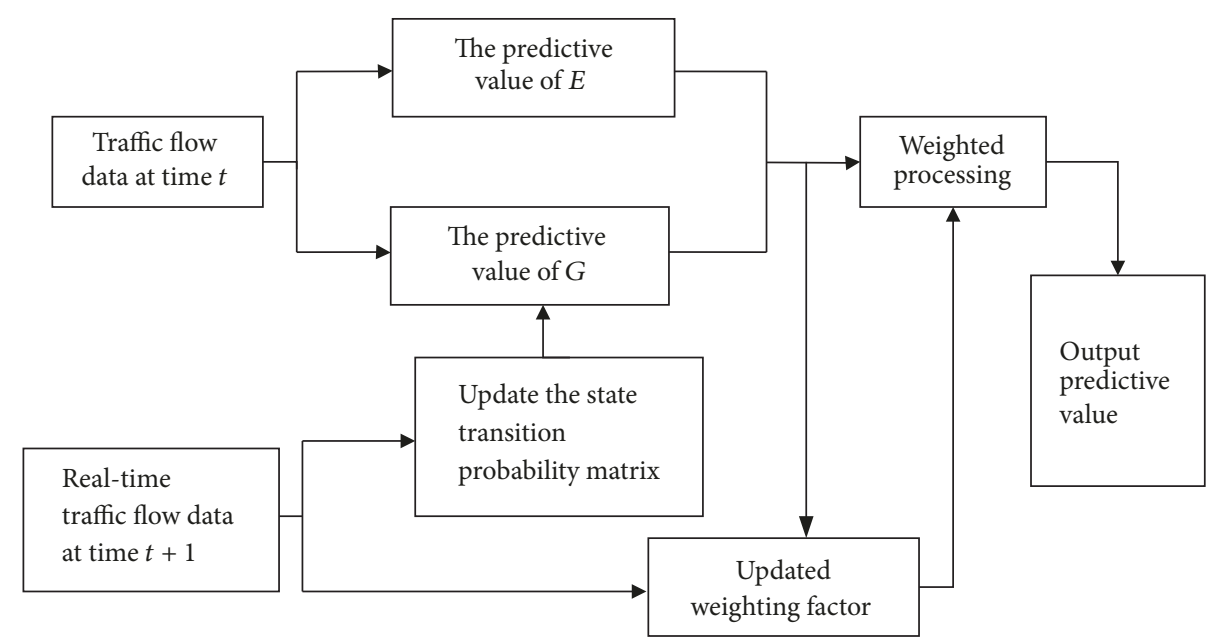

FIGURE 2: Grey difference exponential smoothing forecasting model.

\subsection{Short-Term Flow Traffic Prediction Model Based on Grey} Difference Exponential Smoothing. Exponential smoothing [23] is a widely used sequential prediction algorithm, whose predictive values are obtained by assigning different weights to historical records. Similar to the exponential smoothing algorithm, the first-order difference exponential smoothing algorithm achieves the predicted value by giving different weights to different data sequences. The basic modeling process is shown as follows.

For data sequence (1), the difference sequence is represented as

$$
\nabla \widehat{E}_{t}=x_{t+1}{ }^{(0)}-x_{t}{ }^{(0)}, \quad(t=1,2, \ldots, n-1) .
$$

And the first-order difference exponential smoothing equation is

$$
E_{t+1}=\nabla E_{t}+x_{t}^{(0)}, \quad(t=1,2, \ldots, n),
$$

where $\nabla E_{t}=\alpha \nabla \widehat{E}_{t-1}+(1-\alpha) \nabla E_{t-1}$. Therefore, the forecast result should be

$$
E_{t+1}=\alpha \sum_{j=1}^{t-1}(1-\alpha)^{j-1} \nabla \widehat{E}_{t-j}+(1-\alpha)^{t-1} \nabla E_{1}+x_{t}^{(0)} .
$$

Herein, $0<\alpha<1$ and $(1-\alpha)^{t-1} \nabla E_{1} \rightarrow 0$ when $t \rightarrow \infty$. Thus, formula (17) can be rewritten as

$$
E_{t+1}=\alpha \sum_{j=1}^{t-1}(1-\alpha)^{j-1} \nabla \widehat{E}_{t-j}+x_{t}^{(0)}
$$

We use dynamic weighting factors to weight the models of grey Markov model and first-order difference exponential smoothing model. The predictive value of the grey exponential smoothing model is thus achieved:

$$
Y_{t+1}=\varphi G_{t+1}+\gamma E_{t+1}, \quad(\varphi+\gamma=1) .
$$

In formula (19), $G_{t+1}$ and $E_{t+1}$ are the predictive value of grey Markov and first-order difference exponential smoothing, respectively, while $\varphi$ and $\gamma$ are their corresponding weight factors. The weight factors are calculated as follows:

$$
\begin{aligned}
& \varphi=\frac{\left|x_{t+1}{ }^{(0)}-E_{t+1}\right|+\zeta / 2}{\left|x_{t+1}{ }^{(0)}-G_{t+1}\right|+\left|x_{t+1}{ }^{(0)}-E_{t+1}\right|+\zeta}, \\
& \gamma=\frac{\left|x_{t+1}{ }^{(0)}-G_{t+1}\right|+\zeta / 2}{\left|x_{t+1}{ }^{(0)}-G_{t+1}\right|+\left|x_{t+1}{ }^{(0)}-E_{t+1}\right|+\zeta} .
\end{aligned}
$$

In formula (20), the weight of $\varphi$ and $\gamma$ depends on the absolute value of the difference between the predicted results of different models and the short-term traffic flow. In the meantime, in order to avoid the failure of formula (20), when the two prediction errors are $0, \zeta$ tends to zero. The pseudocode of grey difference exponential smoothing prediction model is shown in Algorithm 1.

According to the synthetic forecasting algorithm, the prediction process of grey difference exponential smoothing prediction model is shown in Figure 2. 
Input: Historical traffic flow data set $x^{(0)}$

Output: Short-term traffic flow forecasting result set $Y$

(1) Initial state probability matrix $P, \alpha, \varphi, \gamma, \xi$

(2) for $t \leftarrow 1$ to $n$ do

(3) Calculate forecasting result $E_{t+1}$ and $G_{t+1}$

(4) $Y_{t+1}=\varphi G_{t+1}+\gamma E_{t+1},(\varphi+\gamma=1)$

(5) Calculate the error $\Delta E_{t+1}$ and $\Delta G_{t+1}$

(6) $\Delta E_{t+1}=\left|x^{(0)}{ }_{t+1}-E_{t+1}\right|, \Delta G_{t+1}=\left|x_{t+1}^{(0)}-G_{t+1}\right|$

(7) Update the weights of $\varphi$ and $\gamma$

(8) $\varphi=\frac{\Delta E_{t+1}+\xi / 2}{\Delta G_{t+1}+\Delta E_{t+1}+\xi}, \gamma=\frac{\Delta G_{t+1}+\xi / 2}{\Delta G_{t+1}+\Delta E_{t+1}+\xi}$

(9) Update the state transition matrix $P$ by $X_{t+1}$

(10) end

(11) return $Y$

Algorithm 1: Pseudocode of grey differential exponent smoothing.

\section{Example and Analysis}

In order to verify the prediction effect of our model, the benchmarks of mean absolute error (MAE), mean square error (MSE), mean absolute percentage error (MAPE), and mean square percentage error (MSPE) are taken as evaluation indexes. Those evaluation indexes are defined as formulas (21)-(24). In those formulas, $X_{t}$ and $\widehat{Y}_{t}$ are the real value and predicted value of the traffic flow at time $t$, respectively, and $n$ stands for the number of samples in the short-term traffic flow.

(1) Mean absolute error is

$$
\mathrm{MAE}=\frac{1}{n} \sum_{t=1}^{n}\left|X_{t}-\widehat{Y}_{t}\right| .
$$

(2) Mean square error is

$$
\mathrm{MSE}=\frac{1}{n} \sqrt{\sum_{t=1}^{n}\left(X_{t}-\widehat{Y}_{t}\right)^{2}} .
$$

(3) Mean absolute percentage error is

$$
\mathrm{MAPE}=\frac{1}{n} \sum_{t=1}^{n}\left|\frac{X_{t}-\widehat{Y}_{t}}{X_{t}}\right| .
$$

(4) Mean square percentage error is

$$
\operatorname{MSPE}=\frac{1}{n} \sqrt{\sum_{t=1}^{n}\left(\frac{X_{t}-\widehat{Y}_{t}}{X_{t}}\right)^{2}} .
$$

The urban expressway in Nan'an District of Chongqing Municipality in China is selected in observation phase to verify the actual prediction effect of the grey difference exponential smoothing model, which is composed of 6 bidirectional lanes. The schematic diagram of the section observation is shown in Figure 3. The data detected include the
TABLE 1: Evaluation index of first-order difference exponential smoothing algorithm.

\begin{tabular}{lcccc}
\hline \multirow{2}{*}{$\alpha$} & \multicolumn{4}{c}{ Index } \\
& MAE & MSE & MAPE & MSPE \\
\hline$\alpha=0.05$ & $\mathbf{2 6 . 8 2 0}$ & $\mathbf{2 . 5 6 3}$ & $\mathbf{0 . 3 8 9}$ & $\mathbf{0 . 0 4 6}$ \\
$\alpha=0.08$ & 27.191 & 2.601 & 0.392 & 0.046 \\
$\alpha=0.10$ & 27.451 & 2.627 & 0.394 & 0.046 \\
$\alpha=0.20$ & 28.828 & 2.760 & 0.408 & 0.047 \\
$\alpha=0.50$ & 33.704 & 3.220 & 0.470 & 0.052 \\
\hline
\end{tabular}

traffic volume, speed, and occupancy of each lane, which are collected via microwave-based vehicle sensors.

The road traffic flow is counted every 5 minutes, and the 226 observation data points of No. 1 Lane are used as the basic data for verification. The fluctuation trend of the data is shown in Figure 4.

As illustrated in Figure 4, there is a strong fluctuation in historical traffic flow, which brought about great difficulties to the prediction. When using the first-order difference exponential smoothing algorithm to predict the short time traffic flow, it is necessary to determine the value of $\alpha$ in the definite formula (18). In this paper, five experiments are carried out according to the parameters listed in Table 1, and the evaluation indexes corresponding to the first-order difference exponential smoothing algorithm are obtained in terms of different benchmarks.

According to the results in Table 1 , when $\alpha=0.05$, the first-order difference exponential smoothing algorithm produces the best effect, and the fitting patterns between the predicted value and actual value are shown in Figure 5.

The initial state probability transfer matrix is calculated from the historical traffic sequence when the grey Markov model is used to predict the short-term traffic flow. The state is divided according to the same interval that every 30 vehicles are used as a state. In the following prediction process, we added real-time traffic data to update Markov state probability transfer matrix in real time. The prediction result of grey Markov model is determined by formula (14), and the fitting effect of predicted value and actual value is shown in Figure 6.

Comparing Figure 6 with Figure 5, the grey Markov model can commendably reflect the trend of traffic flow. However, urban road traffic as a complex nonlinear system shows high uncertainty at different times. The grey Markov chain model cannot accurately predict once sharp fluctuations occur. Meanwhile, compared with the grey Markov chain prediction model, the first-order difference exponential smoothing model is more accordant with the trend of original data, but the prediction results of the algorithm depend on the value of $\alpha$ and, more importantly, are affected by time delays.

Therefore, combining the advantages of grey Markov model and first-order difference exponential smoothing, a new predictive value is obtained by using dynamic weighting factors according to formula (19) and formula (20). The prediction results are shown in Figure 7.

Based on the short-term traffic data of the observed road sections, the test data are predicted, respectively, by formulas 


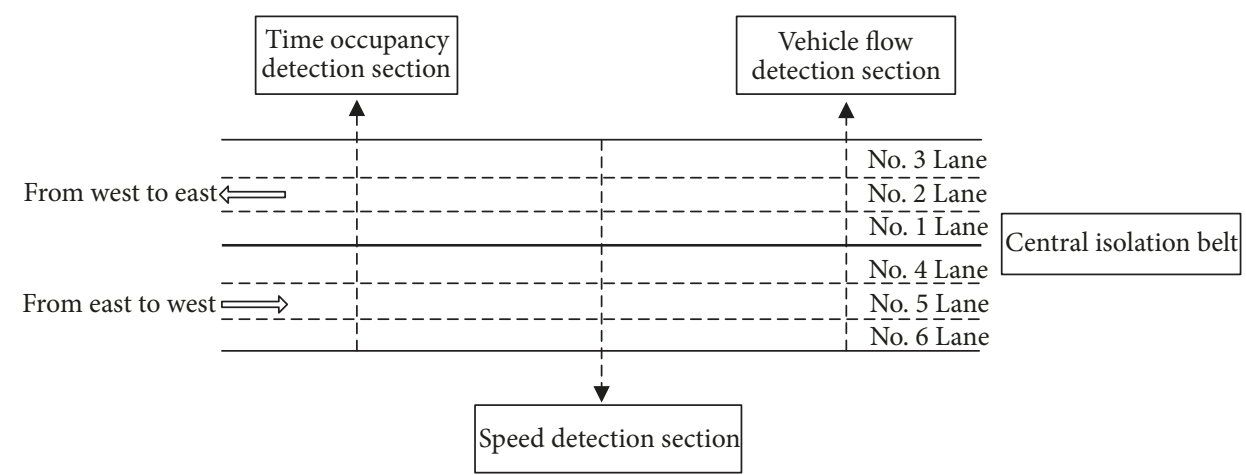

FIGURE 3: Road observation diagram.

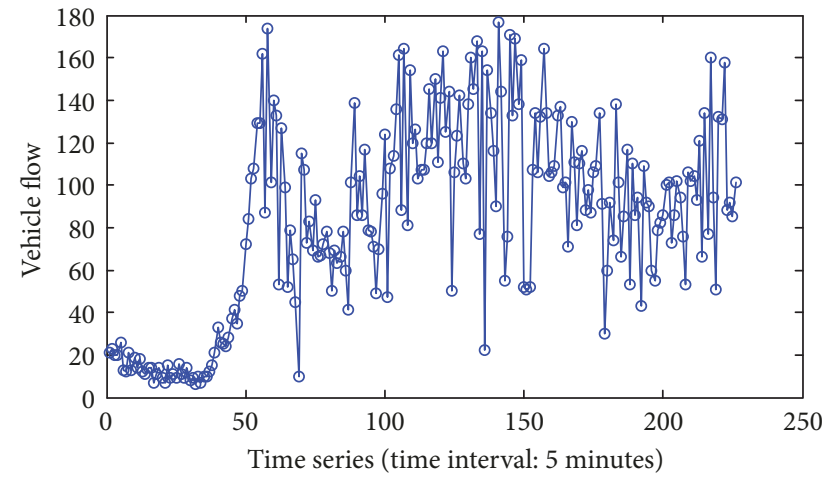

Figure 4: Historical traffic flow data.

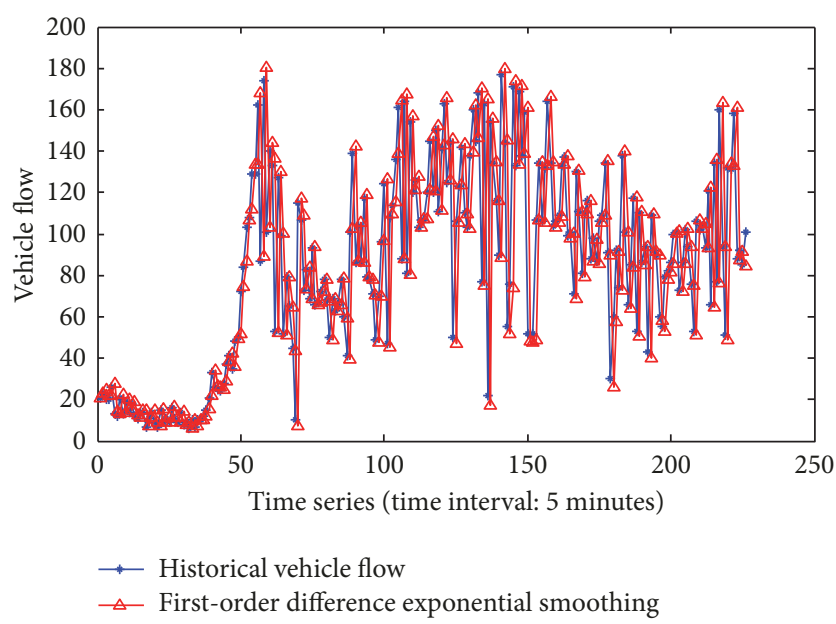

FIGURE 5: Predictive results of first-order difference exponential smoothing model.

(14), (18), and (19). The fitting results of the first 120 data points and the last 106 data points are separately illustrated in Figures 8 and 9.

The evaluation indexes of the above prediction models are shown in Table 2 for the first-order difference exponential smoothing parameter $\alpha=0.05$. Simulation results showed that the grey difference exponential smoothing algorithm can fit short-term traffic flow well and can be applied to urban

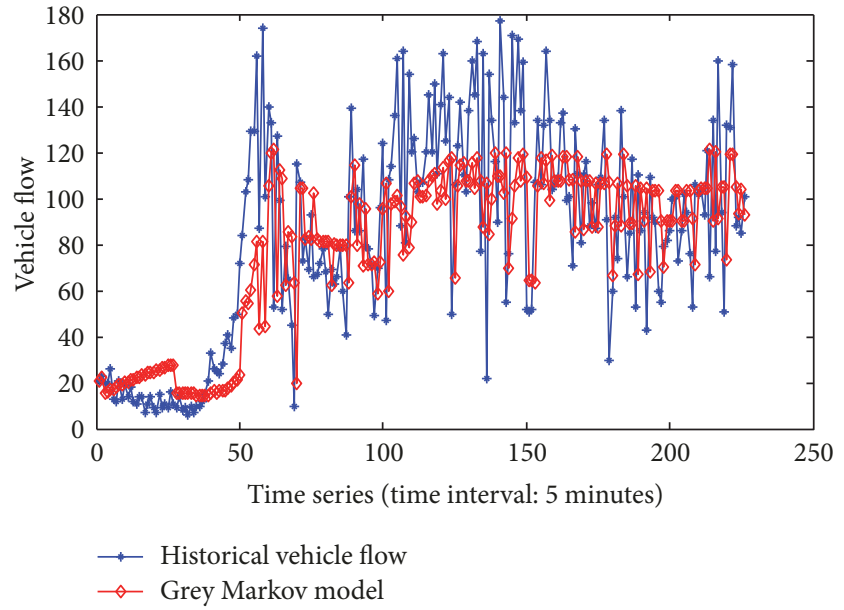

FIGURE 6: Predictive results of grey Markov model.

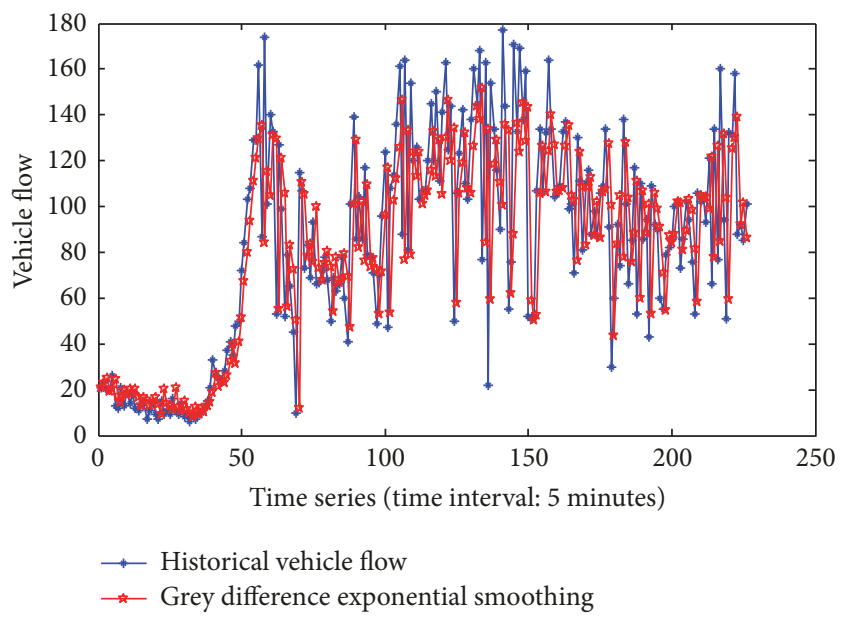

FIGURE 7: Predictive results of grey difference exponential smoothing model.

traffic signal control for short-term traffic flow data with strong fluctuation.

Compared with the above models, the first-order difference exponential smoothing algorithm can closely reflect the 
TABLE 2: Evaluation index of forecasting model.

\begin{tabular}{|c|c|c|c|c|}
\hline \multirow{2}{*}{ Model } & \multicolumn{4}{|c|}{ Index } \\
\hline & MAE & MSE & MAPE & MSPE \\
\hline Grey Markov model & 25.520 & 2.220 & 0.445 & 0.049 \\
\hline First-order difference exponential smoothing model & 26.820 & 2.563 & 0.389 & 0.046 \\
\hline Grey difference exponential smoothing model & 23.943 & 2.237 & 0.368 & 0.043 \\
\hline
\end{tabular}

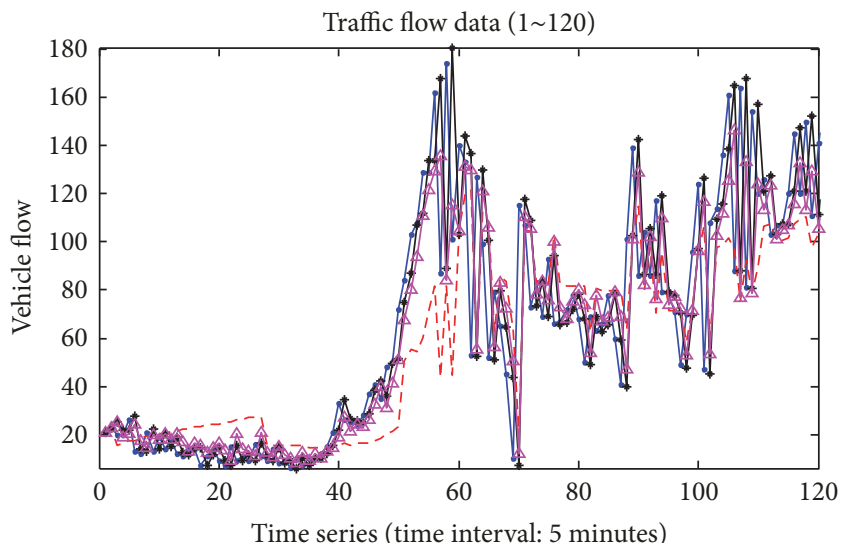

- Historical vehicle flow
-- Grey Markov model
$\rightarrow$ First-order difference exponential smoothing
$\triangle \quad$ Grey difference exponential smoothing

FIGURE 8: Fitting curves between model predictive values and actual test values (the first 120 data points).

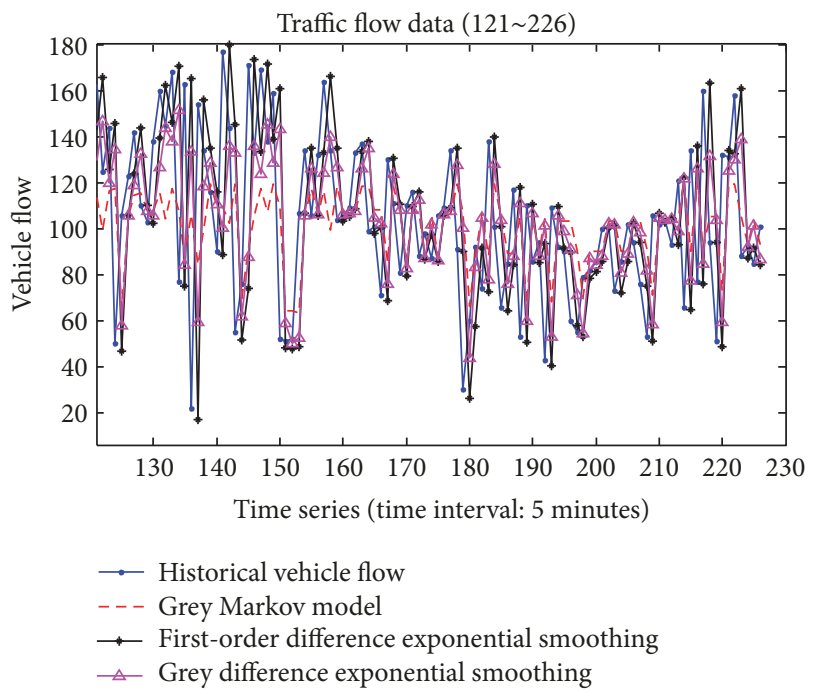

FIGURE 9: Fitting curves between model predictive values and actual test values (the latter 106 data points).

trend of the original data sequence in the prediction trend of the data, but its predicted value depends on the value of $\alpha$ and the trend of the data sequence. For the abrupt data points, the accuracy of its prediction will often be affected to distinct degrees. Compared with the first-order difference exponential smoothing model, the prediction accuracy of grey Markov model considers the development trend of the whole data and the state transition probability of historical traffic flow data. Therefore, the model can reflect the overall equilibrium level for data series with large fluctuation. The grey difference exponential smoothing model combines the advantages of the two models and uses the dynamic weight to obtain the predictive value. The performance evaluation indexes MAE, MSE, MAPE, and MSPE of the improved method are better than the first-order difference exponential smoothing model and grey Markov model for the same traffic flow sequence. However, it can also be seen from Figure 6 and evaluation indexes that the prediction effect of the grey difference exponential smoothing algorithm using dynamic weighted calculation is not absolutely well. Therefore, the method needs to be further optimized and improved.

\section{Conclusion}

This paper mainly contributes to the prediction effect of short-term traffic flow. To overcome the shortcoming of low accuracy in short-term traffic flow prediction caused by strong traffic flow fluctuation, a novel method for short-term traffic forecasting based on the combination of improved grey Verhulst prediction algorithm and first-order difference exponential smoothing is proposed. The main conclusions are as follows.

(1) The grey differential exponential smoothing model combines the advantages of the grey Markov model and the first-order differential exponential smoothing model. The experimental results illustrated that this method is suitable for forecasting short-term traffic flow with large fluctuation. Therefore, this method is practical and feasible.

The analysis shows that this method has smaller prediction error compared with the grey Markov model and the first-order difference exponential smoothing model, and the prediction result is closer to the actual value.

(2) In a word, the method proposed in this paper has obvious advantages. However, due to high uncertainty and nonlinearity of the short-term traffic flow, how to improve the prediction accuracy with fluctuation remains an open problem. So this will be our continuous research orientation.

\section{Conflicts of Interest}

The authors declare that they have no conflicts of interest.

\section{Acknowledgments}

This work is supported by the National Science Foundation of China (NSFC) under Grants 61573076 and 61703063; the Scientific Research Foundation for the Returned Overseas Chinese Scholars under Grant 2015-49; the Program for Excellent 
Talents of Chongqing Higher School under Grant 201418; Science and Technology Research Project of Chongqing Municipal Education Commission of China under Grants KJ1705121 and KJ1705139; the Chongqing Natural Science Foundation of China under Grant CSTC2017jcyjA1665.

\section{References}

[1] Z. Hong-bin, S. Xiao-duan, and H. Yu-long, "Analysis and prediction of comples dynamical characteristics of short-term traffic flow," Acta Physica Sinica, vol. 63, no. 4, Article ID 080502, pp. 51-58, 2014 (Chinese).

[2] D.-R. Huang, J. Song, S.-Q. Li, and H.-Y. Xiang, "Control technology review of traffic congestion in urban road network under networked dynamic scheduling and control," Journal of Traffic and Transportation Engineering, vol. 13, no. 5, pp. 105114, 2013 (Chinese).

[3] S. Oh, Y.-J. Byon, K. Jang, and H. Yeo, "Short-term travel-time prediction on highway: a review on model-based approach," KSCE Journal of Civil Engineering, vol. 3, pp. 1-13, 2017.

[4] N. Fan, X.-M. Zhao, M. Dai, and Y.-S. An, "Short-term traffic flow prediction model," Journal of Traffic and Transportation Engineering, vol. 12, no. 4, pp. 114-119, 2012 (Chinese).

[5] Y.-A. Daraghmi, C.-W. Yi, and T.-C. Chiang, "Negative binomial additive models for short-term traffic flow forecasting in Urban areas," IEEE Transactions on Intelligent Transportation Systems, vol. 15, no. 2, pp. 784-793, 2014.

[6] Y.-S. Jeong, Y.-J. Byon, M. M. Castro-Neto, and S. M. Easa, "Supervised weighting-online learning algorithm for shortterm traffic flow prediction," IEEE Transactions on Intelligent Transportation Systems, vol. 14, no. 4, pp. 1700-1707, 2013.

[7] K. Y. Chan, T. Dillon, E. Chang, and J. Singh, "Prediction of short-term traffic variables using intelligent swarm-based neural networks," IEEE Transactions on Control Systems Technology, vol. 21, no. 1, pp. 263-274, 2013.

[8] M. Lippi, M. Bertini, and P. Frasconi, "Short-term traffic flow forecasting: an experimental comparison of time-series analysis and supervised learning," IEEE Transactions on Intelligent Transportation Systems, vol. 14, no. 2, pp. 871-882, 2013.

[9] N. G. Polson and V. O. Sokolov, "Deep learning for short-term traffic flow prediction," Transportation Research Part C: Emerging Technologies, vol. 79, pp. 1-17, 2017.

[10] H.-H. Xie, X.-H. Dai, and Y. Qi, "Improved K-nearest neighbor algorithm for short-term traffic flow forecasting," Journal of Traffic and Transportation Engineering, vol. 14, no. 3, pp. 87-94, 2014 (Chinese).

[11] X. Xiao, J. Yang, S. Mao, and J. Wen, "An improved seasonal rolling grey forecasting model using a cycle truncation accumulated generating operation for traffic flow," Applied Mathematical Modelling, vol. 51, pp. 386-404, 2017.

[12] L. Lin-chao, H. Shang-lu, and Z. Jian, "Online short-term traffic flow prediction considering the impact of temporal-spatial features," Journal of Transportation Systems Engineering and Information Technology, vol. 16, no. 5, pp. 165-171, 2016 (Chinese).

[13] M. Ma, S. Liang, H. Guo, and J. Yang, "Short-term traffic flow prediction using a self-adaptive two-dimensional forecasting method," Advances in Mechanical Engineering, vol. 9, no. 8, pp. $1-12,2017$

[14] K. Y. Chan, T. S. Dillon, J. Singh, and E. Chang, "Neural-network-based models for short-term traffic flow forecasting using a hybrid exponential smoothing and levenberg-marquardt algorithm," IEEE Transactions on Intelligent Transportation Systems, vol. 13, no. 2, pp. 644-654, 2012.

[15] X. Wang, A. J. Khattak, J. Liu, G. Masghati-Amoli, and S. Son, "What is the level of volatility in instantaneous driving decisions?" Transportation Research Part C: Emerging Technologies, vol. 58, pp. 413-427, 2015.

[16] W. Qing-bo, H. Zhao-cheng, and Z. Xi-shuang, "Prediction of Urban Traffic Performance Index Considering Multiple Factors," Journal of Transportation Systems Engineering and Information Technology, vol. 17, no. 1, pp. 74-81, 2017 (Chinese).

[17] S. Liu, Y. Yang, N. Xie et al., "New progress of grey system theory in the new millennium," The Journal of Grey System, vol. 6, no. 1, pp. 2-31, 2016.

[18] R. Rajesh, V. Ravi, and R. Venkata Rao, "Selection of risk mitigation strategy in electronic supply chains using grey theory and digraph-matrix approaches," International Journal of Production Research, vol. 53, no. 1, pp. 238-257, 2015.

[19] H. Da-rong and H. Li-fen, "Present situation and development of grey system theory in fault forecast application," Journal of Gun Launch \& Control, vol. 3, pp. 88-92, 2009 (Chinese).

[20] D. Song, D. Yao-guo, X. Ning et al., "Optimized background value in grey Verhulst model and its application," Control and Decision, vol. 30, no. 10, pp. 1835-1840, 2015 (Chinese).

[21] Y. Zhang, "Improved background value of grey Verhulst model and its application," System Engineering-Theory and Practice, vol. 33, no. 12, pp. 3168-3171, 2013.

[22] Z. Yi-fan and C. Wen-ying, "Markov chain analysis of metro network in responding to emergency," China Safety Science Journal, vol. 25, no. 2, pp. 165-170, 2015 (Chinese).

[23] Q. Chi and H. Zhong-sheng, "Application of adaptive singexponent smoothing for short-term traffic flow prediction," Control Theory \& Applications, vol. 29, no. 4, pp. 465-469, 2012 (Chinese). 


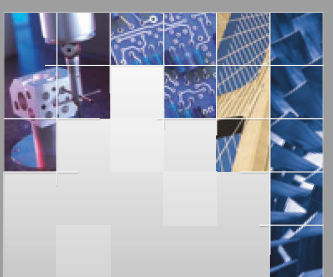

\section{Enfincering}
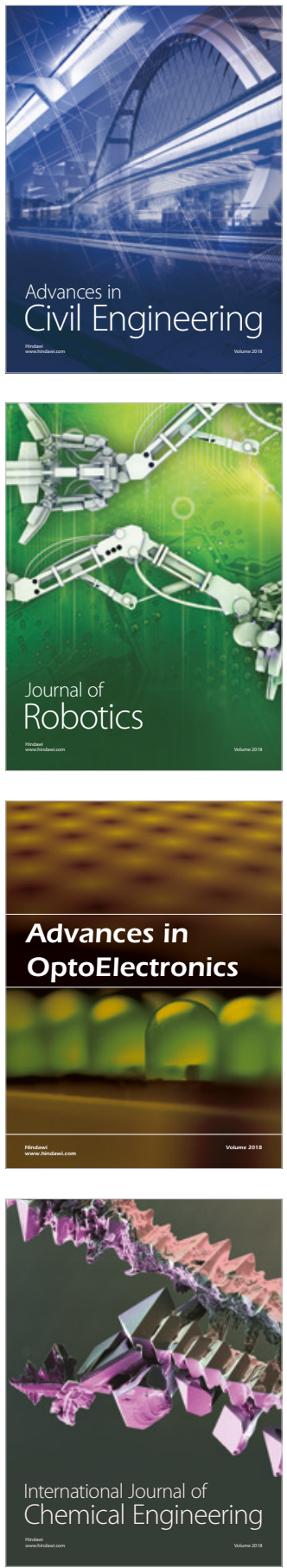

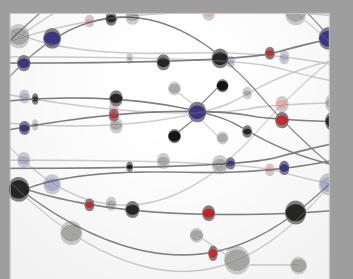

\section{Rotating \\ Machinery}

The Scientific World Journal

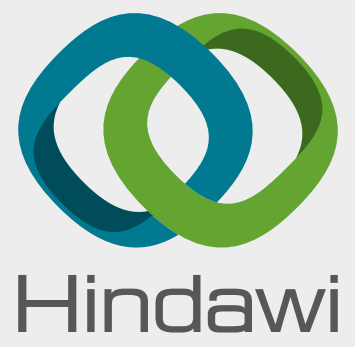

Submit your manuscripts at

www.hindawi.com
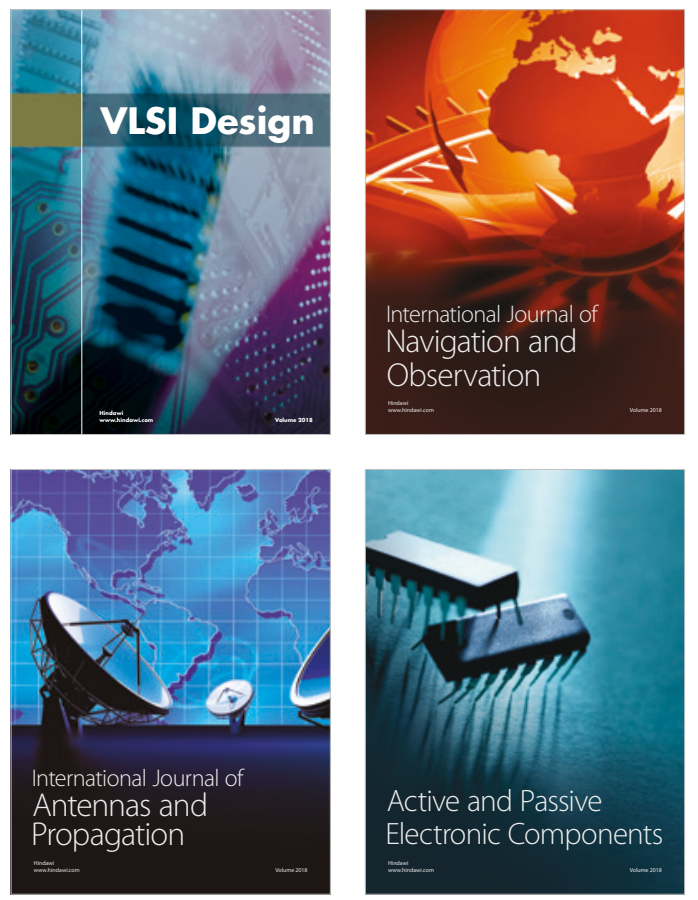
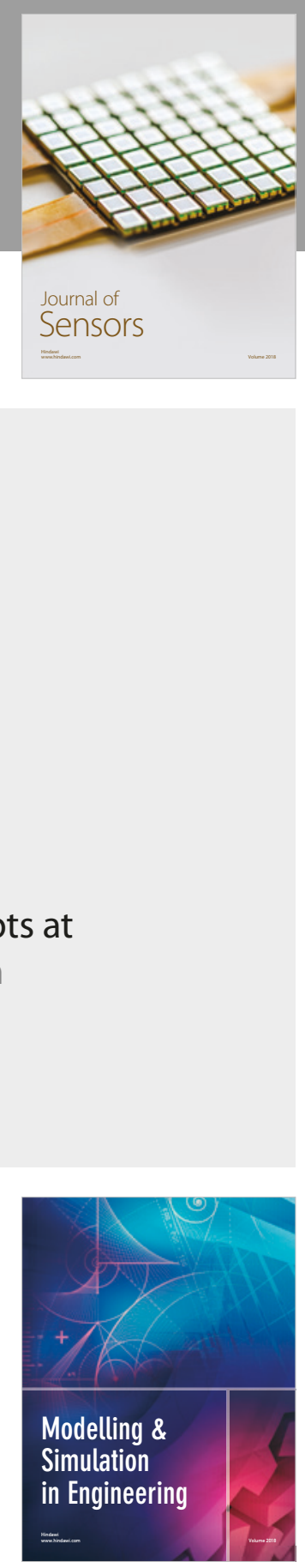

\section{Advances \\ Multimedia}
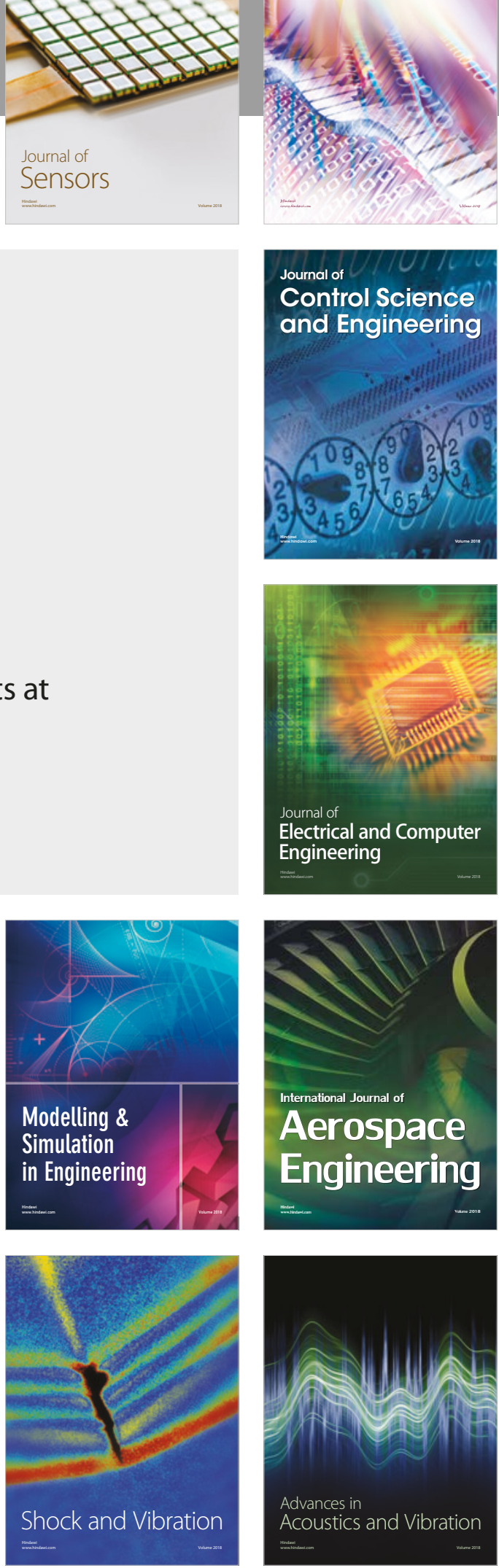